\section{Body MR Imaging at 3 Tesla}

I.R. Kamel and E.M. Merkle, eds.

New York, NY: Cambridge University Press, 2011, 212 pages, $\$ 99$

Three-tesla MRI scanners have been seeing steadily increasing use recently as hardware has matured and pulse sequences have become optimized for a higher field strength. This increase in popularity has been more pronounced for neurologic and musculoskeletal imaging than for body imaging because 3-T MRI with the larger field of view required for the torso tends to be more susceptible to artifacts and energy absorption limits than the imaging of smaller body parts.

The search for higher field strength has been driven by the desire for an increase in signal-to-noise ratio, which can be kept to improve image quality or traded for increased spatial resolution, improved temporal resolution, or both. Little is scientifically proven about the advantage of 3-T over 1.5-T MRI, and thorough knowledge of protocols, physics, and potential pitfalls in 3-T MRI of the body has been limited.

This book is a practical text that provides an in-depth understanding of the differences between imaging at $3 \mathrm{~T}$ and $1.5 \mathrm{~T}$. It explains the physical principles of MRI using a 3-T scanner and the differences between $1.5 \mathrm{~T}$ and $3 \mathrm{~T}$ when applied extracranially. The book's organ-based approach focuses on optimal techniques, providing recommended protocols for the main vendors for 3-T MRI systems. This book, written and edited by experts in the field, guides radiologists in optimizing imaging protocols for 3-T MRI systems, reducing artifacts, and identifying the advantages of using $3 \mathrm{~T}$ in body applications. The intent of this book is to provide a closer look at various MRI applications within the body, with specific emphasis on the effects of a higher 3-T magnetic field strength, protocol optimization, appearance of artifacts, and clinical applications.

There are 14 chapters covering all major thoracic and abdominal organs, including breast, heart, liver, pancreas, gastrointestinal tract, kidney, prostate, and female pelvic organs. Abdominal and pelvic MR angiography and cholangiography are also discussed. Images are clear and instructive, and references are updated.

I highly recommend this book to radiology residents, fellows, and practitioners for understanding the different effects of $1.5-\mathrm{T}$ and 3-T MRI.

E. Edmund Kim

M.D. Anderson Cancer Center

1400 Pressler, Unit 1483

Houston, TX 77030

E-mail: ekim@di.mdacc.tmc.edu

COPYRIGHT @ 2012 by the Society of Nuclear Medicine and Molecular Imaging, Inc.

Published online Jun. 5, 2012

DOI: 10.2967/jnumed.112.105429 